Editorial

\title{
Novel applications of drugs: should we expect more than anesthesia?
}

Volume 8 Issue 2 - 2017

\section{Editorial}

Accumulating data shows effects beyond analgesia and anesthesia for most anesthetic drugs. Owing to the recent advancements in pharmacology, many new-generation anesthetic drugs are now safer than old-generation drugs. The review manuscript entitled: Effects of Anesthesia \& Anesthetic techniques on Cellular Immunity triggered this editorial for this issue of journal of Anesthesia and Critical care. It is crucial for both clinicians and scientists to obtain more information with regard to new insights about drugs commonly used during anesthesia. In other words, identifying the multimodal effect of such drugs can shed some light on their novel applications. A number of biomedical technology management strategies and an emerging pharmaceutical road map have been set forth to explore novel targets in different areas such as cancer, organ protection, immunity etc. Although some contradictory data in subjects with cancer provide a glimpse of the metastatic situation, they can open a new window in the field of regenerative medicine, repurposing them as novel organprotective agents. Many recent scientific achievements are dedicated to biomedicine, which is an excellent combination of engineering and medicine. When it comes to science there are no borders. Several drugs approved by the Food and Drug Administration have been used in accordance with their applications. However, further research may reveal the simultaneous application of such drugs in different organs, and with medical devices. If a specific anesthetic or cardiac drug is effective in pathways such as immunity or bacterial pathogenicity, it can be developed and used in the treatment of some other systemic diseases or used as a topical target in medical devices. As another example in patients with transplantation, we may suggest a specific type of drug not just for its anesthetic or hemodynamic effects but also for its different cellular co-effects.

Recently published data shows multimodal effects of many drugs, for instance, the discussion on the role of lipid emulsion as an organ-protective agent rather than simply an agent used for reversing bupivacaine or other drug toxicities. Searching in literature we can find numerous examples with regard to novel application of different therapeutics including but not limited to: Lipids effect on ischemia reperfusion injury, Propofol anti apoptotic and anti inflammatory effects, Anesthetic anti bacterial effects, novel applications of Calcium Channel blockers, etc. ${ }^{1-14}$ Considering anesthesia as the pillar of patient care during surgeries and in intensive care units that cover the entire body's physiology and pharmacology, should we expect more from the anesthetic drugs?

\section{Acknowledgements}

None.

\section{Conflicts of interest}

The author declares that there is no conflict of interest.

\author{
Negar Motayagheni \\ Department of Anesthesiology, Division of Molecular Medicine, \\ Cardiovascular Research Laboratories, David Geffen School of \\ Medicine at University of California, USA
}

Correspondence: Negar Motayagheni, Anesthesiologist, Regenerative medicine fellowship, Institute for Regenerative Medicine(WFIRM) USA, Email Negar.motayagheni@yahoo.com

Received: May 19, 2017 | Published: May 25, 2017

\section{References}

1. Xu Z, Lu Y, Wang J, et al. The protective effect of propofol against TNF$\alpha$-induced apoptosis was mediated via inhibiting iNOS/NO production and maintaining intracellular $\mathrm{Ca}^{2+}$ homeostasis in mouse hippocampal HT22 cells. Biomed Pharmacother. 2017;91:664-672.

2. Tang QF, Fang ZY, Shi CH. The protective effect and mechanism of sevoflurane on LPS-induced acute lung injury in mice. Am J Transl Res. 2017;9(4):1732-1742.

3. Colucci DG, Puig NR, Hernandez PR. Influence of anaesthetic drugs on immune response: from inflammation to immunosuppression. $O A$ Anaesthetics. 2013;1(3):21.

4. Cruz FF, Rocco PR, Pelosi P. Anti-inflammatory properties of anesthetic agents. Crit Care. 2017;21(1):67.

5. Paola S, Mauro B, Leda G, et al. The Effects of Tramadol and Morphine on Immune Responses and Pain After Surgery in Cancer Patients. Anesth Analg. 2000;90(6):1411-1414.

6. Li J, Ruffenach G, Kararigas G, et al. Intralipid protects the heart in late pregnancy against ischemia/reperfusion injury via Caveolin2/STAT3/ GSK-3beta pathway. J Mol Cell Cardiol. 2017;102: 108-116.

7. Li J, Motayagheni N, Barakati N, et al. Intralipid Protects the Heart in Late Pregnancy Against Ischemia/Reperfusion Injury by Reducing Cardiomyocyte Apoptosis via Mir122 Induction. Circulation Research. 2017;119(Suppl 1):A442-A442

8. Motayagheni N, Eghbali M. Complete Reversal of Xylazine-induced Bradycardia with Intralipid in Female Mice. Circulation Research. 2016;119(Suppl 1):A253-A253

9. Motayagheni N, Phan S, Eshraghi C, et al. Inhibition Of Leptin Receptor Abolishes Intralipid-induced Cardioprotection against IschemiaReperfusion Injury. Cardiology. 2016;134:241.

10. Motayagheni N, Eghbali M. Reversal of Xylazine-induced Bradycardia with Intralipid. Cardiology. 2016;134:431.

11. Motayagheni N. From Bupivacaine to Intralipid: Leading Edge. J Anesth Clin Res. 2016;4(6):00164. 
12. Li J, Motayagheni N, Barakati N, et al. Intralipid Protects the Heart Against Ischemia/Reperfusion Injury by Reducing Cardiomyocyte Apoptosis via miR122 Induction in Late Pregnancy. Cardiology. 2016;134:313.

13. Motayagheni N, Sharma S, Li J, et al. Implication of miR-1 and miR-144 in Intralipid-induced Cardioprotection against Ischemia/Reperfusion Injury. Cardiology. 2016;134:430.

14. Aamri E, Basnawi A. Effects of Anesthesia \& Anesthetic techniques on Cellular Immunity. J Anesth Crit Care Open Access. 2017;(6):00283. 